\title{
Soil fauna communities and microbial activities response to litter and soil properties under degraded and restored forests of Hyrcania
}

\author{
Maryam Bazyari ${ }^{(1)}$, \\ Vahid Etemad ${ }^{(2)}$, \\ Yahya Kooch ${ }^{(3)}$, \\ Anoushirvan Shirvany ${ }^{(2)}$
}

(1) Department of Forestry and Forest Economy, Faculty of Natural Resources, University of Tehran, Tehran (Iran); (2) Faculty of Natural Resources, University of Tehran, Tehran (Iran); (3) Faculty of Natural Resources \& Marine Sciences, Tarbiat Modares University, 46417-76489, Noor, Mazandaran (Iran)

@ Vahid Etemad (vetemad@ut.ac.ir)

Received: Jul 12, 2020 - Accepted: Sep 06, 2021

Citation: Bazyari M, Etemad V, Kooch Y, Shirvany A (2021). Soil fauna communities and microbial activities response to litter and soil properties under degraded and restored forests of Hyrcania. iForest 14: 490-498. doi: 10.3832/ifor3583-014 [online 2021-1111]

Communicated by: Maurizio Ventura

Reforestation has long been the best practice to restore degraded forests due to human interventions. In this paper we investigated the effect of forest degradation (DNF) along with reforestation using 4 endemic species (Alnus subcordata, ASP; Acer velutinum, AVP; Cupressus sempervirens, CSP; Quercus castaneifolia Mey, QCP) on forest's soil chemical and biological indicators compared to a close-to-virgin natural forest (VNF). For this study, a total of 24 physico-chemical and 25 biological and microbial indicators were measured in soils of all 6 forest stands along with the litter properties. Results showed that the lowest soil quality was observed under DNF, CSP, and QCP which was the result of forest cover degradation in DNF and low litter quality, especially low $\mathrm{pH}$ and high $\mathrm{C}: \mathrm{N}$, in CSP and QCP. Soil fauna communities were significantly affected by tree species. We found two times higher density of earthworms in VNF compared to ASP, but in DNF the density was 5 times lower than VNF. We found no epigeic earthworms in QCP, CSP and DNF and no endogeic earthworms in DNF. Acarina and Collembola density was high in VNF and ASP, but they showed significant differences (VNF>ASP), and their density sharply decreased in other stands, especially in CSP ( 3 times lower than VNF) and DNF (8 to 10 times lower than VNF). Nematode density was statistically equal in VNF, ASP, and AVP, but significantly lower in other stands. Protozoa, bacteria and fungi densities were significantly higher in VNF and ASP (VNF>ASP) compared to each other and other forest stands. Basal respiration, substrate induced respiration, microbial biomass $\mathrm{N}$ and $\mathrm{P}$, and carbon availability index was also higher in VNF and ASP compared to other stands. Although VNF has the best condition because of old forest cover and high diversity, ASP soil showed significant improvements, demonstrating the importance of litter quality in soil restoration. Restoration effectiveness ranking of the four tested species on soil improvement are therefore ASP $>A V P>Q C P>C S P$. The significant improvement of soil quality under ASP compared to other reforestated stands, only after 3 decades, emphasizes the importance of tree species selection and litter quality on soil chemical and biological restoration.

Keywords: Forest Restoration, Reforestation, Litter Quality, Soil Biological Activity, Soil Chemical Properties, Soil Fauna

\section{Introduction}

Reforestation of clear-cut forests and agricultural lands is a well known practice to restore natural forest ecosystems around the world. As soil functional traits (especially carbon sequestration) are affected by forest degradation, the process of restoration can lead to significant increase in the ecological value of the temperate forests and in soil quality (Sanji et al. 2020), and improves the microbial community (MC) of the forest soils (Vázquez et al. 2020). Although reforestation, due to low diversity in tree species, does not lead to the same soil and ecosystem characteristics as of natural forests (Kooch et al. 2020), these stands are the most closed ecosystems to the natural ones that human are able to establish, and at some points, the ecosystem and soil characteristics could also be close to natural ones (Sanji et al. 2020). Therefore, reforestation has impacts on several aspects, both aboveground and belowground.
At a global scale, reforestation is currently one of the strategies to tackle climate change, as tree plantations can be exploited to sequester atmospheric carbon (Nave et al. 2019). Recent studies found that large scale reforestations, after the bare soil $C$ efflux to the atmosphere, can lead to significant increase in soil carbon pools. This means that the reforestation of clear-cut forests and abandoned agricultural lands are among the best options to increase belowground carbon sequestration (Józefowska et al. 2017).

At the local scale, forest restoration can lead to significant increase in biodiversity of soil fauna and flora, however, at a lower diversity compared to natural forests. Soil fauna play an important role in soil development and layer formation. Studies have shown that fauna activity have the potential to completely exploit soil organic layer (Oe) and develop a thick organomineral layer (A) in forest soil (Frouz et al. 2013). Other studies showed that soil microbial 
and fauna activities, especially earthworms, can significantly modify chemical and microbial properties of the forest soils through the digestion of organic matter in their guts. Therefore, soil properties can be affected by a combination of tree species and soil fauna activities (Frouz 2018). On the other hand, reforestation has significant effects on soil MC structure and consequently, can lead to increased soil $\mathrm{C}$ sequestration and soil nitrogen content (Shao et al. 2019). MC of the forest soils is also influenced by the quantity and chemical properties of the input litter. Shao et al. (2019) found that in the early stages of reforestation, MC have a significant effect on SOC accumulation, however, at the mature stages, these communities will be affected by vegetation diversity and litter quality. Therefore, there is a close mutual relationship between soil MC and tree species characteristics in natural and restored forests (Tajik et al. 2020).

Studies also found several influential factors on soil chemical properties under natural restored forests. While soil degradation due to deforestation has significant negative effects on chemical soil properties (Sanji et al. 2020), reforestation has a positive effect on soil quality according to the selected tree species (Wang et al. 2019). Tree species not only improve soil chemical quality through litter, but also through root exudates. Several studies have shown that reforestation using coniferous species can significantly increase SOC (Vázquez et al. 2020), while introducing native broadleaved species will result in higher quality and SOC chemical stability (Wang et al. 2019), with higher enzyme activity in the soil (Diao et al. 2020). Therefore, tree species selection for reforestation and forest restoration is a key influential factor in forest management.

As reforestation using different tree spe cies may not lead to final ecosystem characteristics matching those of the natural ones, monitoring the effect of restoration practices on the ecosystem (especially soil) under different reforestation strategies is crucial. Here we examined soil chemical and biological response to different reforestation species after forest clear-cut. We aimed at determining which of four different tree species can lead to closer soil characteristics to that of a natural forest. The goals of this study are: (i) determining litter quality among different reforestation species; (ii) understanding the relationship between soil quality and biological and $\mathrm{mi}$ crobial soil characteristics in degraded and restored forests; (iii) determining whole species effect on soil chemical and biological characteristics of the restored forest soil; and (iv) disentangling the relationship between soil fauna community changes, soil carbon/nitrogen/phosphorus, and litter quality under different tree species.

\section{Materials and methods}

\section{Site description}

The study site has an area of 1394 ha and is located in the city of Ramsar (Mazandaran province, northern Iran - 36 $46^{\circ} 37^{\prime \prime}$ and $36^{\circ} 51^{\prime} 00^{\prime \prime} \mathrm{N}, 50^{\circ} 43^{\prime} 10^{\prime \prime}$ and $51^{\circ} 35^{\prime} 00^{\prime \prime}$ $\mathrm{E})$. The site has an altitudinal range of 60$2130 \mathrm{~m}$ a.s.l. with an average slope of $40 \%$. The climate is classified as seasonal temperate humid with an average annual rainfall of $773 \mathrm{~mm}$ and temperature of $12.3^{\circ} \mathrm{C}$. The monthly average temperature ranges from 5.2 in February to $19.5^{\circ} \mathrm{C}$ in July, without frost periods. According to the USDA Soil Taxonomy, the forest soils are classified as Loamy-Clay-Sand Alfisols, developed on conglomerate composed of lime stones belonging to the Jurassic period (Soil Survey Staff 2002).

Significant parts of these natural forests have been severely damaged during past decades. After clear cutting in 1988, the Forests and Rangelands Organization of Iran (FROI) performed reforestation practices to restore these habitats. Reforestation were done using Alder (Alnus subcordata C.A. Mey.) in an area of 16 ha, Maple (Acer velutinum Boiss.) 11 ha, Oak (Quercus castaneifolia C.A. Mey.) 4 ha, Mediterranean cedar (Cupressus sempervirens var. horizontalis) 8 ha. Degraded forests dominant species includes hornbeam (Carpinus betulus L.) and ironwood (Parrotia persica C.A. Mey.) species with an area of approximately 6 ha, and close-to-virgin natural forest (VNF) includes Oak (Quercus castaneifolia C.A. Mey.), hornbeam (Carpinus betulus L.) and ironwood (Parrotia persica C.A. Mey.), with an area of approximately $10 \mathrm{ha}$.

\section{Sampling}

Six forest types were selected for the purpose of this study, which includes pure reforestation of Alnus subcordata (ASP), Acer velutinum (AVP), Quercus castaneifolia (QCP), Cupressus sempervirens plantation (CSP), a close-to-virgin natural forest (VNF, oak-hornbeam-ironwood as control) and a degraded forest (DNF, hornbeam and ironwood). All the selected forest types were close to each other with similar soil texture and topographic conditions. A 4 ha area $(200 \times 200 \mathrm{~m})$ was selected in each site for sampling. In order to reduce the boundary effects, the rows around the sampling area were not considered. Sampling was performed during summer 2019 using four parallel transects $(200 \mathrm{~m}$ in length and $66 \mathrm{~m}$ apart from each other) and five soil and litter samples $(25 \times 25 \mathrm{~cm})$ were randomly collected at a depth of 0-10 cm (Fig. 1). A total of 60 samples (i.e., 5 litter samples and 5 soil samples type) were transferred to the laboratory for litter and soil analysis (Kooch \& Bayranvand 2019).

\section{Laboratory analyses}

Litter thickness was measured using a tape, and then transported in bags to the laboratory, washed gently for 30 seconds to remove mineral soil, and dried at $70{ }^{\circ} \mathrm{C}$ for $48 \mathrm{~h}$, and then the dry weight measured. Dried litter samples were finely grounded and analyzed. Total C and N, along with nutrient contents in litter samples were determined using dry combustion with an elemental analyzer (Fisons
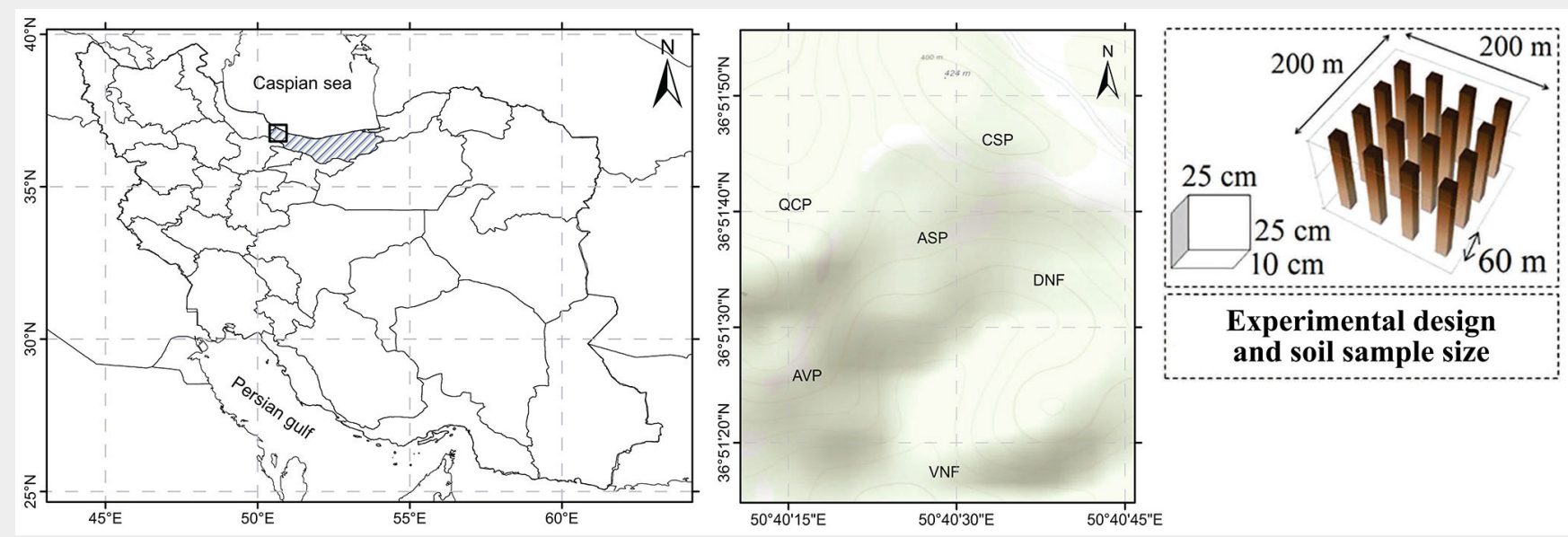

Fig. 1 - Locations of the studies forest stands and sampling plots. A 4-ha square was randomly selected in each stand (right panel) and 5 out of 16 subplots were selected at random to measure litter and soil properties. 
EA1108, Milan, Italy), calibrated by the BBOT [2.5-bis-(5-tert-butyl-benzoxazol-2-yl) -thiophen] standard (ThermoQuest Italia S.p.A., Rodano, MI, Italy). Litter phosphorus (LP) concentration was determined spectrophotometrically. An atomic absorption spectrophotometer was used to determine total litter potassium (LK), calcium (LCa), and magnesium (LMg) concentration by flame emission.

A portion of soil samples was stored in polyethylene bags for biological analysis at $4{ }^{\circ} \mathrm{C}$ until processed. Another portion was air-dried and passed through 2-mm sieve (aggregates were broken to pass through a $2 \mathrm{~mm}$ sieve). Bulk density (BD) was measured by the clod method (Plaster 2013). Soil texture was determined using the Bouyoucos hydrometric method (Bouyoucos 1962). Soil water content was measured by drying soil samples at $105{ }^{\circ} \mathrm{C}$ for $24 \mathrm{~h}$. Soil $\mathrm{pH}$ and electric conductivity (EC) were measured using an Orion Ionalyzer Model $901 \mathrm{pH}$ meter in a 1:2.5 (soil: water) solution (Tavakoli et al. 2018).

Soil organic $C$ was measured using the Walkey-Black method (Allison 1965) and total $\mathrm{N}$ using a semi Micro-Kjeldahl method (Bremner \& Mulvaney 1982). Available P was measured by a spectrophotometer using the Olsen method (Homer \& Pratt 1961), and available $\mathrm{K}, \mathrm{Ca}$, and $\mathrm{Mg}$ were determined using an atomic absorption spectrophotometer (by ammonium acetate extraction at $\mathrm{pH}$ 9). Particulate organic matter carbon (POM-C) and nitrogen (POM-N) were determined by physical fractionation (Handayani et al. 2010). Fine roots (diameter $<2 \mathrm{~mm}$ ) were collected from each soil sample and dried at $70^{\circ} \mathrm{C}$. The earthworms were collected simultaneously with the soil sampling by hand sorting, and identified using the Edwards et al. (2021) key based on ecological categories (i.e., epigeic, anecic and endogeic) by external characteristics. Earthworm biomass was then determined after drying at $60{ }^{\circ} \mathrm{C}$ for $24 \mathrm{~h}$ and weighing. Soil Acarina and Collembola were extracted with the help of modified Tullgren funnel, as described by Hutson \& Veitch (1987). Nematodes were extracted from $100 \mathrm{~g}$ soil sample (fresh weight) by a modified cotton-wool filter method (Liang et al. 2009). Following the extraction method, soil protozoa population densities were counted under a microscope (Mayzlish \& Steinberger 2004). Soil total bacteria and fungi were counted following extraction from soil (10 g fresh weight were mixed with $90 \mathrm{ml}$ distilled water) using the homogenization and centrifugation techniques and culturing as described by Wollum (1983). Soil basal respiration (BR) was determined by measuring the $\mathrm{CO}_{2}$ evolved in a 3-day incubation experiment at $25^{\circ} \mathrm{C}$ (Alef 1995). Substrate induced respiration (SIR), was determined using $1 \%$ glucose solution as substrate and the evolved $\mathrm{CO}_{2}$ was measured after $72 \mathrm{~h}$ incubation. The evolved $\mathrm{CO}_{2}$ was adsorbed in $\mathrm{NaOH}$ and measured by $\mathrm{HCl}$ titration (Anderson \&
Domsch 1990). Carbon, nitrogen and phosphorous in the microbial biomass (MBC, $M B N$, and $M B P$, respectively) were measured by fumigation-extraction method (Brookes et al. 1985). The soil metabolic quotient $\left(\mathrm{qCO}_{2}=\mathrm{BR} / \mathrm{MBC}-\right.$ Anderson \& Domsch 1990) and carbon availability index (CAI = BR/SIR -Cheng et al. 1996) were calculated based on the values of organic $C$, $B R$, substrate induced respiration (SIR) and $M B C$.

\section{Statistical analysis}

The normality of the variables was tested by the Kolmogorov-Smirnov test, and Levene's test was used to examine the homogeneity of variances. Data are presented as mean \pm standard error throughout the text, figures and tables. One-way analysis of variance (ANOVA) was used to compare litter and soil features data among the different land covers. Duncan test was further employed to test for mean differences with $\alpha=0.05$. Relationships between the measured parameters was also investigated using Pearson's correlation. All the
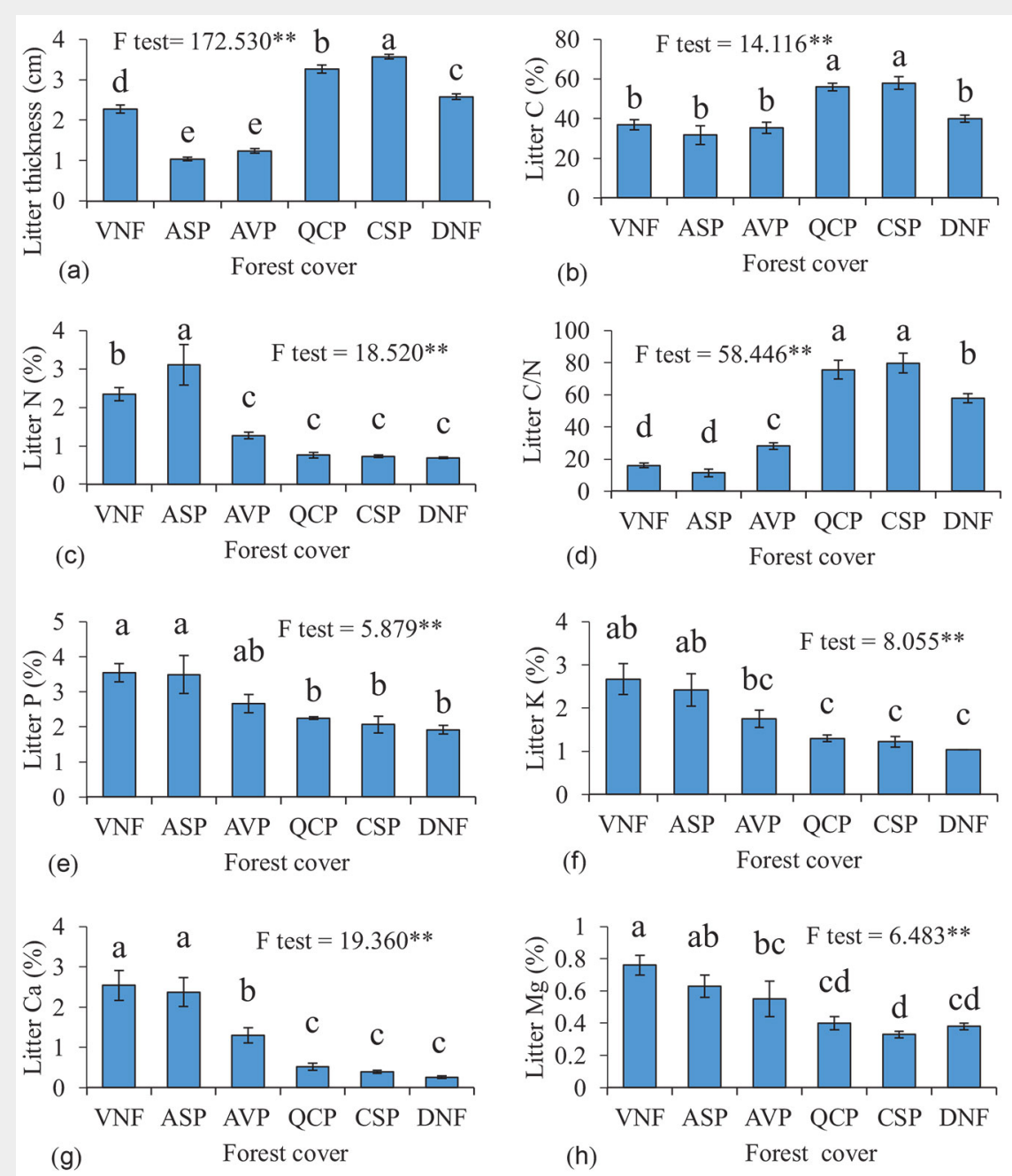

Fig. 2 - Mean values of the litter properties across different forest covers. Different letters indicate significant differences $(p<0.05)$ between the means of forest covers after Duncan test. (VNF): close-to-virgin natural forest; (ASP): Alnus subcordata plantations; (AVP): Acer velutinum; (QCP): Quercus castaneifolia; (CSP): Cupressus sempervirens; (DNF): degraded natural forest. Error bars represent the standard error. 
Tab. 1 - Mean ( \pm standard error, SE) of the soil physical, chemical and biological properties analyzed under different forest covers. (VNF): virgin natural forest; (ASP): Alnus subcordata plantation; (AVP:): Acer velutinum; (QCP): Quercus castaneifolia; (CSP): Cupressus sempervirens; (DNF): degraded natural forest. Different letters indicate significant differences $(p<0.05)$ between the means in different forest covers after Duncan test.

\begin{tabular}{|c|c|c|c|c|c|c|c|c|}
\hline \multirow[t]{2}{*}{ Soil Properties } & \multicolumn{8}{|c|}{ Forest cover } \\
\hline & VNF & ASP & AVP & QCP & CSP & DNF & F-test & P-value \\
\hline Bulk density $\left(\mathrm{g} \mathrm{cm}^{-3}\right)$ & $1.43 \pm 0.04^{\mathrm{b}}$ & $1.51 \pm 0.07^{\mathrm{b}}$ & $1.46 \pm 0.06^{\mathrm{b}}$ & $1.06 \pm 0.01^{c}$ & $1.03 \pm 0.00^{c}$ & $1.73 \pm 0.01^{\mathrm{a}}$ & 37.732 & $<0.001$ \\
\hline Sand (\%) & $25.00 \pm 2.36^{b c}$ & $19.80 \pm 2.57^{c}$ & $34.20 \pm 8.40^{\mathrm{ab}}$ & $27.40 \pm 2.99^{\mathrm{abc}}$ & $29.40 \pm 4.61^{\mathrm{abc}}$ & $40.20 \pm 2.13^{a}$ & 2.603 & 0.051 \\
\hline Silt (\%) & $39.40 \pm 1.77^{\mathrm{ab}}$ & $46.40 \pm 4.92^{\mathrm{a}}$ & $30.20 \pm 3.77^{\mathrm{b}}$ & $43.60 \pm 2.31^{\mathrm{a}}$ & $45.00 \pm 4.72^{\mathrm{a}}$ & $36.80 \pm 2.35^{\mathrm{ab}}$ & 2.969 & 0.032 \\
\hline Clay (\%) & $35.60 \pm 1.24^{\mathrm{a}}$ & $33.80 \pm 3.39^{\mathrm{a}}$ & $35.60 \pm 5.67^{\mathrm{a}}$ & $29.00 \pm 2.04^{\mathrm{ab}}$ & $25.60 \pm 3.23^{\mathrm{ab}}$ & $23.00 \pm 0.63^{b}$ & 2.892 & 0.035 \\
\hline Water content (\%) & $48.48 \pm 3.05^{a}$ & $33.65 \pm 3.39^{b c}$ & $38.61 \pm 4.92^{\mathrm{abc}}$ & $44.73 \pm 3.91^{\mathrm{ab}}$ & $45.59 \pm 3.79^{a}$ & $30.86 \pm 2.47^{c}$ & 3.714 & 0.012 \\
\hline $\mathrm{pH}\left(1: 2.5 \mathrm{H}_{2} \mathrm{O}\right)$ & $7.06 \pm 0.11^{\mathrm{a}}$ & $7.12 \pm 0.06^{a}$ & $6.88 \pm 0.16^{\mathrm{ab}}$ & $6.32 \pm 0.09^{b c}$ & $6.29 \pm 0.52^{b c}$ & $5.77 \pm 0.09^{c}$ & 5.243 & 0.002 \\
\hline $\mathrm{EC}\left(\mathrm{ds} \mathrm{m}^{-1}\right)$ & $0.30 \pm 0.01^{\mathrm{a}}$ & $0.32 \pm 0.03^{\mathrm{a}}$ & $0.24 \pm 0.01^{\mathrm{a}}$ & $0.17 \pm 0.01^{\mathrm{b}}$ & $0.15 \pm 0.04^{\mathrm{b}}$ & $0.12 \pm 0.00^{\mathrm{b}}$ & 9.962 & $<0.001$ \\
\hline Organic C (\%) & $4.29 \pm 0.45^{\mathrm{bc}}$ & $3.44 \pm 0.24^{c}$ & $3.96 \pm 0.43^{\mathrm{bc}}$ & $5.09 \pm 0.65^{b}$ & $6.62 \pm 0.26^{\mathrm{a}}$ & $3.91 \pm 0.35^{\mathrm{bc}}$ & 7.311 & $<0.001$ \\
\hline Total N (\%) & $0.46 \pm 0.05^{\mathrm{a}}$ & $0.56 \pm 0.11^{\mathrm{a}}$ & $0.27 \pm 0.02^{b}$ & $0.22 \pm 0.03^{b}$ & $0.17 \pm 0.04^{b}$ & $0.12 \pm 0.01^{b}$ & 9.071 & $<0.001$ \\
\hline $\mathrm{C} / \mathrm{N}$ ratio & $9.29 \pm 0.13^{\mathrm{cd}}$ & $7.23 \pm 1.46^{d}$ & $14.60 \pm 0.66^{\mathrm{cd}}$ & $23.20 \pm 2.37^{b c}$ & $46.48 \pm 8.83^{\mathrm{a}}$ & $33.84 \pm 7.01^{\mathrm{ab}}$ & 10.388 & $3<0.001$ \\
\hline Available $\mathrm{P}\left(\mathrm{mg} \mathrm{kg}^{-1}\right)$ & $29.21 \pm 0.86^{\mathrm{a}}$ & $27.15 \pm 2.13^{a}$ & $19.34 \pm 3.11^{\mathrm{b}}$ & $13.51 \pm 1.67^{c}$ & $11.03 \pm 2.06^{c}$ & $10.49 \pm 0.57^{c}$ & 17.932 & $<0.001$ \\
\hline Available $\mathrm{K}\left(\mathrm{mg} \mathrm{kg}^{-1}\right)$ & $415.80 \pm 16.08^{\mathrm{a}}$ & $405.20 \pm 52.78^{\mathrm{a}}$ & $316.60 \pm 21.05^{\mathrm{b}}$ & $184.20 \pm 13.97^{c}$ & $166.00 \pm 19.86^{c}$ & $155.60 \pm 18.61^{c}$ & 19.764 & $4<0.001$ \\
\hline Available $\mathrm{Ca}\left(\mathrm{mg} \mathrm{kg}^{-1}\right)$ & $293.80 \pm 28.35^{a}$ & $277.20 \pm 42.95^{\mathrm{ab}}$ & $214.60 \pm 16.33^{b}$ & $101.40 \pm 16.50^{c}$ & $97.20 \pm 7.97^{c}$ & $89.00 \pm 6.72^{c}$ & 16.342 & $<0.001$ \\
\hline Available $\mathrm{Mg}\left(\mathrm{mg} \mathrm{kg}^{-1}\right)$ & $79.00 \pm 1.76^{a}$ & $71.60 \pm 5.83^{\mathrm{a}}$ & $65.60 \pm 8.44^{\mathrm{ab}}$ & $50.80 \pm 7.35^{\mathrm{bc}}$ & $38.60 \pm 3.23^{c}$ & $42.60 \pm 3.35^{c}$ & 8.780 & $<0.001$ \\
\hline POM-C $\left(\mathrm{g} \mathrm{kg}^{-1}\right)$ & $3.61 \pm 0.20^{\mathrm{ab}}$ & $2.13 \pm 0.20^{c}$ & $2.75 \pm 0.51^{\mathrm{bc}}$ & $3.76 \pm 0.45^{\mathrm{a}}$ & $4.44 \pm 0.18^{\mathrm{a}}$ & $1.02 \pm 0.17^{d}$ & 14.987 & $<0.001$ \\
\hline POM-N ( $\left.\mathrm{g} \mathrm{kg}^{-1}\right)$ & $0.50 \pm 0.05^{b}$ & $0.67 \pm 0.04^{\mathrm{a}}$ & $0.27 \pm 0.02^{c}$ & $0.15 \pm 0.01$ & $0.12 \pm 0.00^{d}$ & $0.07 \pm 0.01^{\mathrm{d}}$ & 62.340 & $<0.001$ \\
\hline
\end{tabular}

was significantly higher in CSP (57\%) compared to other forest covers. QCP was the second forest cover with the highest litter $C(56 \%)$ and the lowest litter $C$ was observed in ASP (31.68\%). On the contrary, establishment of ASP led to an increase in the concentration of litter N (3.11\%), which was significantly higher than other studied forest covers. Litter $\mathrm{C} / \mathrm{N}$ ratio in different forest covers was significantly different with the lowest value in ASP, which was the closest one to VNF (11.42 and 15.96, respectively). VNF had the highest concentration of $\mathrm{Ca}, \mathrm{P}, \mathrm{K}$, and $\mathrm{Mg}$, but no significant difference was observed between VNF and ASP (Fig. 2).

Significant difference was also found between different forest covers in terms of soil physical and chemical properties. Bulk density in DNF (1.73 $\left.\mathrm{gr} \mathrm{cm}^{-3}\right)$ was significantly higher compared to all other forest covers, and the lowest bulk density was observed in CSP $\left(1.03 \mathrm{gr} \mathrm{cm}^{-3}\right)$ and QCP $\left(1.06 \mathrm{gr} \mathrm{cm}^{-3}\right)$. Soil texture showed significant difference among some forest covers. ASP had the lowest sand content (19.8\%) while DNF had the highest (40.20\%). Silt content was only significantly different between ASP and AVP, while clay showed significantly lower content in DNF compared to VNF, ASP, and AVP (Tab. 1).

Soil water content in VNF and CSP (48.48\% and $45.59 \%$, respectively) was significantly higher than in DNF. Soil pH also showed significantly low value in DNF (5.77), while ASP and VNF showed the highest values. Soil organic carbon content (SOC) and $\mathrm{C} / \mathrm{N}$ ratio were significantly different among the sites, with CSP having the highest value (6.62\% and $46.48 \%)$. Soil total $\mathrm{N}$, and available $\mathrm{P}, \mathrm{K}$ and $\mathrm{Mg}$ were sig- nificantly higher in VNF and ASP compared to other forest covers, while the highest amount of available soil Ca (293.80 $\mathrm{mg} \mathrm{kg}^{-1}$ ) was observed under VNF. Lowest soil total $\mathrm{N}$, and available $\mathrm{P}$, and $\mathrm{K}$ were observed in DNF.

POM-C and POM-N showed similar pattern to that of soil organic $\mathrm{C}$ and total $\mathrm{N}$ with the highest average POM-C of $4.44 \pm$ 0.18 in CSP and POM-N of $0.67 \pm 0.04$ in ASP (Tab. 1).

\section{Soil fauna and microbial biomass}

The highest fine root biomass was observed in VNF $\left(62.58 \mathrm{~g} \mathrm{~m}^{-2}\right)$ while the lowest was measured in DNF (11.9 $\left.\mathrm{g} \mathrm{m}^{-2}\right)$ soils. According to our data, degradation of natural forests resulted in a significant decrease in soil fauna, to the extent that reforestation could not restore this community close to that of VNF. VNF showed 7.18 $\mathrm{mg} \mathrm{m}^{-2}$ biomass of epigeic earthworms, while no biomass was detected in QCP, CSP, and DNF soils; yet, the differences between the stands were non-significant. Anecic earthworm biomass and density were also lower in CSP and AVP compared to other stands and they were absent in DNF, while we detected $9.7 \mathrm{mg} \mathrm{m}^{-2}$ in VNF. Endogeic earthworm biomass was significantly higher in VNF $\left(24.84 \mathrm{mg} \mathrm{m}^{-2}\right)$ compared to AVP (8.63 $\left.\mathrm{mg} \mathrm{m}^{-2}\right)$, QCP (4.49 mg $\left.\mathrm{m}^{-2}\right), \operatorname{CSP}\left(4.65 \mathrm{mg} \mathrm{m}^{-2}\right)$, and DNF $(1.26 \mathrm{mg}$ $\mathrm{m}^{-2}$ ). Earthworm density and biomass in VNF $\left(3.20 \mathrm{n} \mathrm{m}^{-2}\right.$, and $41.72 \mathrm{mg} \mathrm{m}^{-2}$, respectively) were significantly higher compared to other stands and more than two folds higher than ASP stand. Earthworm population was close to zero in each DNF and we found only one earthworm in $5 \mathrm{~m}^{-2}$ (at a depth of $10 \mathrm{~cm}$ ) of forest soils in DNF.
Acarina and Collembola density showed three different levels. Both of these organisms showed significantly higher density in VNF compared to other stands with averages of 47,032 and 37,088 organisms per $\mathrm{m}^{2}$, respectively. Also, in ASP the density of both organisms were significantly higher than the remaining stands (39,560 for Acarina and 26,502 for Collembola per $\mathrm{m}^{2}$ ). Acarina density was similar in AVP, QCP, CSP, and DNF, while Collembola was the lowest in DNF, with 10 times lower density compared to VNF. Soil nematode community was recovered in ASP (250.6 in $100 \mathrm{~g}$ of soil) and AVP (220.4 in $100 \mathrm{~g}$ of soil) to a level that is not significantly different from VNF (279 in $100 \mathrm{~g}$ of soil), while protozoa density was significantly higher in VNF $\left(646.2 \times 10^{2} \mathrm{~g}\right.$ soil $)$ and then ASP $\left(468.8 \times 10^{2}\right.$ $\mathrm{g}$ soil) compared to other stands. Protozoa community was 7 times higher in VNF compared to DNF ( $89.4 \times 10^{2} \mathrm{~g}$ soil $)$.

Total bacteria and total fungi in ASP (4.11 and $2.45 \times 10^{7} \mathrm{~g}$ soil, respectively) were close to VNF ( 4.87 and $3.63 \times 10^{7} \mathrm{~g}$ soil, respectively); however, the differences were significant at $p<0.05$. Both communities were more than 10 times higher in VNF compared to DNF ( 0.41 and $0.33 \times 10^{7} \mathrm{~g}$ soil, respectively). Bacterial community was statistically equal in AVP $\left(1.95 \times 10^{7} \mathrm{~g}\right.$ soil $)$ compared to QCP $\left(1.88 \times 10^{7} \mathrm{~g}\right.$ soil), and CSP ( $0.67 \times 10^{7} \mathrm{~g}$ soil) compared to DNF (0.41 $\times 10^{7} \mathrm{~g}$ soil), while fungal community was similar in AVP (0.76 $\times 10^{7} \mathrm{~g}$ soil), QCP $(0.72$ $\times 10^{7} \mathrm{~g}$ soil $)$, CSP $\left(0.57 \times 10^{7} \mathrm{~g}\right.$ soil $)$, and DNF $\left(0.33 \times 10^{7} \mathrm{~g}\right.$ soil - Tab. 2$)$.

\section{Soil biological activity}

Soil basal and substrate induced respiration (BR and SIR) in VNF and ASP were not 
Tab. 2 - Mean ( \pm SE) of the soil biological properties analyzed under different forest covers. (VNF): virgin natural forest; (ASP): Alnus subcordata plantation; (AVP:): Acer velutinum; (QCP): Quercus castaneifolia; (CSP): Cupressus sempervirens; (DNF): degraded natural forest. Different letters indicate significant differences $(p<0.05)$ between the means in different forest covers after Duncan test.

\begin{tabular}{|c|c|c|c|c|c|c|c|c|}
\hline \multirow{2}{*}{ Soil properties } & \multicolumn{8}{|c|}{ Forest cover } \\
\hline & VNF & ASP & AVP & QCP & CSP & DNF & F-test & P-value \\
\hline Fine root biomass $\left(\mathrm{g} \mathrm{m}^{-2}\right)$ & $62.58 \pm 5.32^{\mathrm{a}}$ & $42.72 \pm 6.07^{b}$ & $37.57 \pm 2.13^{b}$ & $33.59 \pm 5.66^{\mathrm{b}}$ & $29.57 \pm 3.42^{b}$ & $11.09 \pm 0.73^{c}$ & 14.936 & $<0.001$ \\
\hline Epigeic density $\left(\mathrm{n} \mathrm{m}^{-2}\right)$ & $0.40 \pm 0.24^{\mathrm{a}}$ & $0.20 \pm 0.20^{\mathrm{a}}$ & $0.20 \pm 0.20^{\mathrm{a}}$ & $0.00 \pm 0.00^{\mathrm{a}}$ & $0.00 \pm 0.00^{\mathrm{a}}$ & $0.00 \pm 0.00^{\mathrm{a}}$ & 1.143 & 0.365 \\
\hline Epigeic biomass $\left(\mathrm{mg} \mathrm{m}^{-2}\right.$ ) & $7.18 \pm 4.43^{\mathrm{a}}$ & $2.31 \pm 2.31^{\mathrm{a}}$ & $1.93 \pm 1.93^{\mathrm{a}}$ & $0.00 \pm 0.00^{\mathrm{a}}$ & $0.00 \pm 0.00^{\mathrm{a}}$ & $0.00 \pm 0.00^{\mathrm{a}}$ & 1.626 & 0.191 \\
\hline Anecic density $\left(\mathrm{n} \mathrm{m}^{-2}\right)$ & $0.80 \pm 0.37^{\mathrm{a}}$ & $0.20 \pm 0.20^{\mathrm{a}}$ & $0.20 \pm 0.20^{\mathrm{a}}$ & $0.40 \pm 0.24^{\mathrm{a}}$ & $0.20 \pm 0.20^{\mathrm{a}}$ & $0.00 \pm 0.00^{\mathrm{a}}$ & 1.425 & 0.251 \\
\hline Anecic biomass ( $\mathrm{mg} \mathrm{m}^{-2}$ ) & $9.69 \pm 4.86^{\mathrm{a}}$ & $2.65 \pm 2.65^{\mathrm{a}}$ & $2.65 \pm 2.65^{\mathrm{a}}$ & $7.95 \pm 4.87^{\mathrm{a}}$ & $2.33 \pm 2.33^{\mathrm{a}}$ & $0.00 \pm 0.00^{\mathrm{a}}$ & 1.256 & 0.315 \\
\hline Endogeic density $\left(\mathrm{n} \mathrm{m}^{-2}\right)$ & $2.00 \pm 0.31^{\mathrm{a}}$ & $1.00 \pm 0.31^{\mathrm{ab}}$ & $0.80 \pm 0.37^{b}$ & $0.60 \pm 0.60^{\mathrm{b}}$ & $0.40 \pm 0.40^{\mathrm{b}}$ & $0.20 \pm 0.20^{\mathrm{b}}$ & 2.711 & 0.044 \\
\hline Endogeic biomass ( $\mathrm{mg} \mathrm{m}^{-2}$ ) & $24.84 \pm 4.13^{\mathrm{a}}$ & $13.18 \pm 4.92^{\mathrm{ab}}$ & $8.63 \pm 3.85^{b}$ & $4.49 \pm 4.49^{\mathrm{b}}$ & $4.65 \pm 4.65^{b}$ & $1.26 \pm 1.26^{\mathrm{b}}$ & 4.410 & 0.005 \\
\hline Earthworm density $\left(\mathrm{n} \mathrm{m}^{-2}\right)$ & $3.20 \pm 0.73^{\mathrm{a}}$ & $1.40 \pm 0.40^{\mathrm{b}}$ & $1.20 \pm 0.48^{\mathrm{b}}$ & $1.00 \pm 0.57^{\mathrm{b}}$ & $0.60 \pm 0.60^{b}$ & $0.20 \pm 0.20^{\mathrm{b}}$ & 3.961 & 0.009 \\
\hline $\begin{array}{l}\text { Earthworm biomass } \\
\left(\mathrm{mg} \mathrm{m}^{-2}\right)\end{array}$ & $41.72 \pm 9.73^{a}$ & $18.15 \pm 4.79^{b}$ & $13.22 \pm 4.73^{\mathrm{b}}$ & $12.44 \pm 5.10^{b}$ & $6.99 \pm 6.99^{b}$ & $1.26 \pm 1.26^{\mathrm{b}}$ & 5.450 & 0.002 \\
\hline Acarina density $\left(\mathrm{n} \times 10^{3} \mathrm{~m}^{-2}\right)$ & $47.03 \pm 5.53^{\mathrm{a}}$ & $39.56 \pm 0.93^{b}$ & $13.00 \pm 2.10^{c}$ & $11.46 \pm 0.44^{c}$ & $10.77 \pm 0: 20^{c}$ & $5.82 \pm 1.35^{c}$ & 47.780 & $<0.001$ \\
\hline 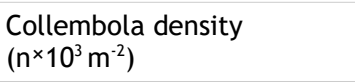 & $37.09 \pm 4.03^{\mathrm{a}}$ & $26.50 \pm 3.76^{\mathrm{b}}$ & $16.72 \pm 1.77^{c}$ & $13.27 \pm 1.30^{c}$ & $12.19 \pm 0.27^{c}$ & $3.51 \pm 0.68^{\mathrm{d}}$ & 23.674 & $<0.001$ \\
\hline $\begin{array}{l}\text { Total nematodes } \\
\text { (in } 100 \mathrm{~g} \text { soil) }\end{array}$ & $279.00 \pm 28.82^{\mathrm{a}}$ & $250.60 \pm 42.83^{a}$ & $220.40 \pm 31.87^{a}$ & $102.60 \pm 15.69^{b}$ & $84.00 \pm 7.14^{b}$ & $35.40 \pm 9.46^{\mathrm{b}}$ & 14.920 & $<0.001$ \\
\hline $\begin{array}{l}\text { Protozoa density } \\
\left(\times 10^{2} \text { g soil }\right)\end{array}$ & $646.20 \pm 123.42^{\mathrm{a}}$ & a $468.80 \pm 67.10^{b}$ & $249.80 \pm 38.66^{c}$ & $163.40 \pm 15.59^{c}$ & $110.80 \pm 4.65^{c}$ & $89.40 \pm 1.43^{c}$ & 13.887 & $<0.001$ \\
\hline Total bacteria $\left(\times 10^{7} \mathrm{~g}\right.$ soil $)$ & $4.87 \pm 0.23^{\mathrm{a}}$ & $4.11 \pm 0.12^{b}$ & $1.95 \pm 0.19^{c}$ & $1.88 \pm 0.28^{c}$ & $0.67 \pm 0.11^{d}$ & $0.41 \pm 0.09^{d}$ & 92.089 & $<0.001$ \\
\hline Total fungi $\left(\times 10^{7} \mathrm{~g}\right.$ soil $)$ & $3.63 \pm 0.23^{\mathrm{a}}$ & $2.45 \pm 0.22^{b}$ & $0.76 \pm 0.07^{c}$ & $0.72 \pm 0.07^{c}$ & $0.57 \pm 0.07^{c}$ & $0.33 \pm 0.02^{c}$ & 86.421 & $<0.001$ \\
\hline
\end{tabular}

statistically different, with averages of 0.76 and $1.64 \mathrm{mg} \mathrm{CO}_{2} \mathrm{~g}^{-1}$ day $^{-1}$ for VNF and 0.73 and $1.49 \mathrm{mg} \mathrm{CO}_{2} \mathrm{~g}^{-1}$ day $^{-1}$ for ASP, respectively, but they were significantly higher than those of other forest covers. Soil carbon microbial biomass showed the highest value $\left(600.61 \mathrm{mg} \mathrm{kg}^{-1}\right)$ in CSP, which was significantly higher than those of ASP, AVP and DNF, but statistically equals to VNF and QCP. Nitrogen microbial biomass was significantly higher in ASP (48.07 $\mathrm{mg} \mathrm{kg}^{-1}$ ) and VNF (41.48 $\left.\mathrm{mg} \mathrm{kg}^{-1}\right)$, compared to other forest covers, while phosphorus microbial biomass was the highest in VNF $(81.40 \mathrm{mg}$ $\mathrm{kg}^{-1}$ ). Further, microbial biomass indicators were significantly the lowest in DNF.

The ratios $M B C / M B N$ and $M B C / M B P$ were significantly higher in CSP (33.95 and 22.31) compared to other forest covers, while MBN/MBP was similar for all forest covers $(p>0.05)$. Metabolic quotient ratio in ASP (1.88 $\mu \mathrm{g} \mathrm{CO}_{2}-\mathrm{C} \mathrm{mg}^{-1} \mathrm{MBC}$ day-1) was significantly higher than in other sites, and five times higher than that of $\operatorname{CSP}\left(0.37 \mu \mathrm{g} \mathrm{CO}_{2}\right.$ -
C mg-1 MBC day ${ }^{-1}$ ), but not significantly dif ferent from DNF. Finally, ASP and VNF showed the highest carbon availability index (CAI), which was significantly higher compared to other forest stands ( 0.49 and 0.48 , respectively - Tab. 3 ).

The studied forest sites and their litter and soil characteristics were then analyzed using PCA. The first and second axes accounted for $52.43 \%$ and $14.55 \%$ of the total variance, respectively. The first PC axis discriminates fairly well two groups of forest

Tab. 3 - Mean ( \pm SE) of the soil microbial properties analyzed under different forest covers. (VNF): virgin natural forest; (ASP): Alnus subcordata plantation; (AVP:): Acer velutinum; (QCP): Quercus castaneifolia; (CSP): Cupressus sempervirens; (DNF): degraded natural forest. Different letters indicate significant differences $(p<0.05)$ between the means in different forest covers after Duncan test.

\begin{tabular}{|c|c|c|c|c|c|c|c|}
\hline \multirow{2}{*}{ Soil properties } & \multicolumn{7}{|c|}{ Forest cover } \\
\hline & VNF & ASP & AVP & QCP & CSP & DNF $\quad F$ test & P-value \\
\hline $\begin{array}{l}\text { Basal respiration } \\
\left(\mathrm{mg} \mathrm{CO}_{2} \mathrm{~g}^{-1} \text { day }^{-1}\right)\end{array}$ & $0.76 \pm 0.03^{a}$ & $0.73 \pm 0.05^{\mathrm{a}}$ & $0.35 \pm 0.02^{b}$ & $0.26 \pm 0.02^{b c}$ & $0.22 \pm 0.01^{c}$ & $0.12 \pm 0.01^{d} 74.678$ & $<0.001$ \\
\hline $\begin{array}{l}\text { Substrate induced respi- } \\
\text { ration }\left(\mathrm{mg} \mathrm{CO}_{2} \mathrm{~g}^{-1} \text { day }^{-1}\right)\end{array}$ & $1.64 \pm 0.14^{\mathrm{a}}$ & $1.49 \pm 0.10^{\mathrm{a}}$ & $1.10 \pm 0.04^{b}$ & $1.08 \pm 0.01^{\mathrm{b}}$ & $1.00 \pm 0.05^{b}$ & $0.84 \pm 0.07^{b} 13.056$ & $<0.001$ \\
\hline $\begin{array}{l}\text { Microbial biomass carbon } \\
\left(\mathrm{mg} \mathrm{kg}^{-1}\right)\end{array}$ & $504.02 \pm 72.62^{\mathrm{ab}}$ & $415.93 \pm 57.29^{b}$ & $417.39 \pm 54.61^{\mathrm{b}}$ & $519.05 \pm 69.75^{\mathrm{ab}}$ & $600.61 \pm 14.63^{\mathrm{a}}$ & $111.78 \pm 28.7^{c} \quad 9.913$ & $<0.001$ \\
\hline $\begin{array}{l}\text { Microbial biomass } \\
\text { nitrogen }\left(\mathrm{mg} \mathrm{kg}^{-1}\right)\end{array}$ & $41.48 \pm 5.66^{\mathrm{a}}$ & $48.07 \pm 3.77^{\mathrm{a}}$ & $26.03 \pm 2.48^{\mathrm{b}}$ & $21.37 \pm 0.66^{\mathrm{b}}$ & $18.22 \pm 1.30^{\mathrm{b}}$ & $6.21 \pm 1.02^{c} 25.725$ & $<0.001$ \\
\hline $\begin{array}{l}\text { Microbial biomass } \\
\text { phosphorous (mg kg-1) }\end{array}$ & $81.40 \pm 0.97^{a}$ & $60.20 \pm 7.78^{b}$ & $37.00 \pm 3.84^{c}$ & $30.00 \pm 3.17^{c}$ & $27.20 \pm 1.35^{c}$ & $11.20 \pm 0.86^{\mathrm{d}} 43.373$ & $<0.001$ \\
\hline $\mathrm{MBC} / \mathrm{MBN}$ & $12.60 \pm 1.81^{\mathrm{cd}}$ & $8.88 \pm 1.37^{d}$ & $16.33 \pm 2.22^{\mathrm{bcd}}$ & $24.20 \pm 3.20^{\mathrm{b}}$ & $33.95 \pm 3.54^{\mathrm{a}}$ & $18.56 \pm 4.55^{\mathrm{bc}} 8.969$ & $<0.001$ \\
\hline $\mathrm{MBC} / \mathrm{MBP}$ & $6.17 \pm 0.87^{c}$ & $7.38 \pm 1.21^{c}$ & $12.25 \pm 2.51^{\mathrm{bc}}$ & $17.43 \pm 2.23^{\mathrm{ab}}$ & $22.31 \pm 1.26^{\mathrm{a}}$ & $10.24 \pm 2.81^{c} \quad 9.978$ & $<0.001$ \\
\hline MBN/MBP & $0.51 \pm 0.06^{\mathrm{a}}$ & $0.84 \pm 0.11^{\mathrm{a}}$ & $0.77 \pm 0.17^{\mathrm{a}}$ & $0.75 \pm 0.08^{\mathrm{a}}$ & $0.67 \pm 0.05^{\mathrm{a}}$ & $0.54 \pm 0.06^{\mathrm{a}} \quad 1.639$ & 0.188 \\
\hline $\begin{array}{l}\text { Metabolic quotient } \\
\left(\mu \mathrm{g} \mathrm{CO} \mathrm{CO}_{2}-\mathrm{C} \mathrm{mg}^{-1} \mathrm{MBC} \text { day }^{-1}\right)\end{array}$ & $1.73 \pm 0.41^{\mathrm{ab}}$ & $1.88 \pm 0.27^{\mathrm{a}}$ & $0.92 \pm 0.16^{\mathrm{abc}}$ & $0.56 \pm 0.09^{b c}$ & $0.37 \pm 0.03^{c}$ & $1.72 \pm 0.73^{\mathrm{ab}} 3.152$ & 0.025 \\
\hline Carbon availability index & $0.48 \pm 0.05^{\mathrm{a}}$ & $0.49 \pm 0.02^{\mathrm{a}}$ & $0.32 \pm 0.02^{\mathrm{b}}$ & $0.24 \pm 0.02^{\mathrm{b}}$ & $0.23 \pm 0.01^{\mathrm{bc}}$ & $0.15 \pm 0.01^{c} 20.924$ & $<0.001$ \\
\hline
\end{tabular}




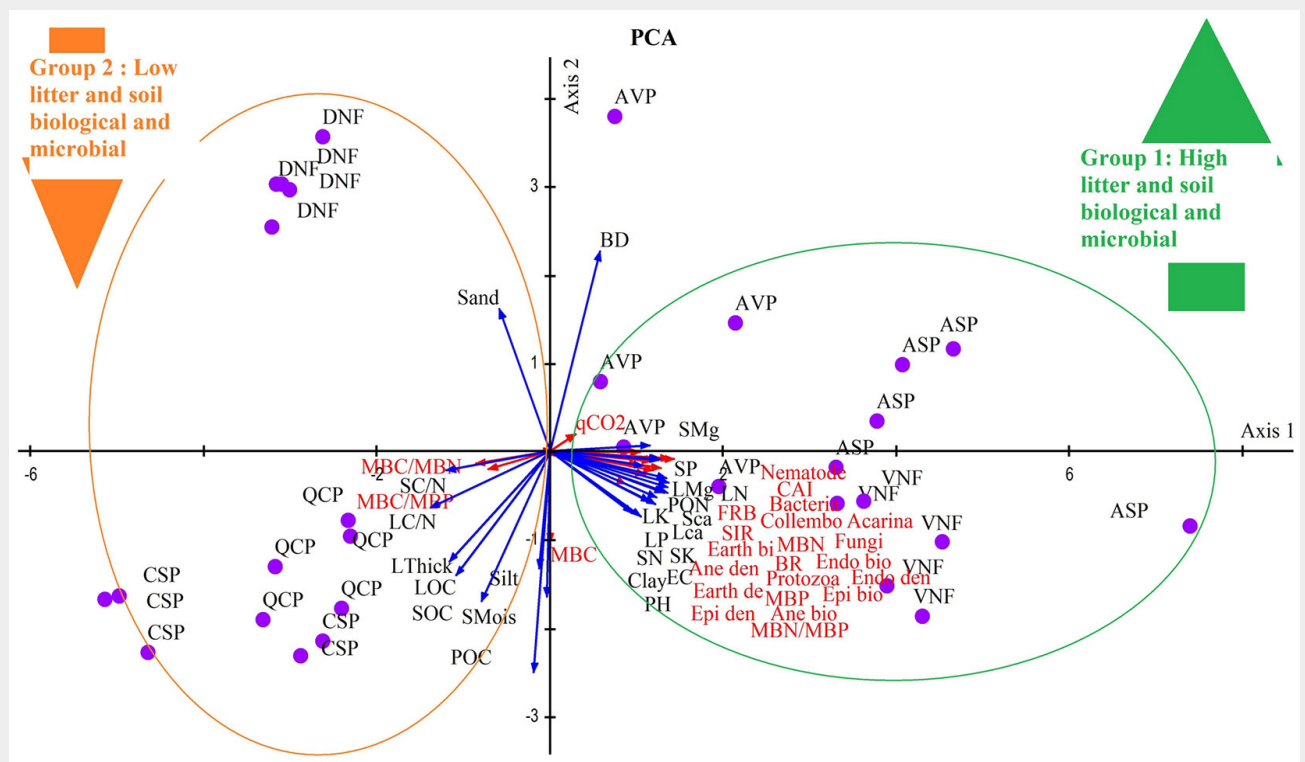

Fig. 3 - PCA analysis of the measured variables in different forest covers. The first PCA axis accounted for $52.43 \%$ of the total variance, while the second axis explained $14.55 \%$.

covers (sites). The first group reflects good the measured variables have positive corlitter quality, accumulation of nutrients, relation, however some key variables (e.g., and more biological and microbial activity, litter $\mathrm{C} / \mathrm{N}$ ratio and thickness and soil $\mathrm{C} / \mathrm{N}$ and is mainly formed by VNF and ASP sites. ratio) have significant negative correlation While the second group along the PC1 axis with most of the measured variables. Litter shows low quality of litter and soil, low nu- calcium content is likely a key parameter trients and low biological activity at DNF, significantly affecting soil chemical properCSP, and QCP sites (Fig. 3).

\section{Relationship between soil properties}

Correlation analysis of the studied soil and litter properties showed that most of ties and fauna communities in a positive way, while soil potassium and phosphorus have significant correlation with soil microfauna communities. Potassium and phosphorus also have significant correlation with soil biological activity. Acarina, Collembola, Nematodes, Protozoa, Bacteria, and Fungi have significant correlation with soil biological properties, especially soil respiration and CAI. It seems that MBC only has significant correlation with soil carbon content (SOC, POM-C, FRB); however, soil bulk density has significant negative relationship with this parameter. Soil metabolic quotient is strongly correlated with the ratios of $\mathrm{MBC} / \mathrm{MBN}$ and $\mathrm{MBC} / \mathrm{MBP}$ (Fig. 4).

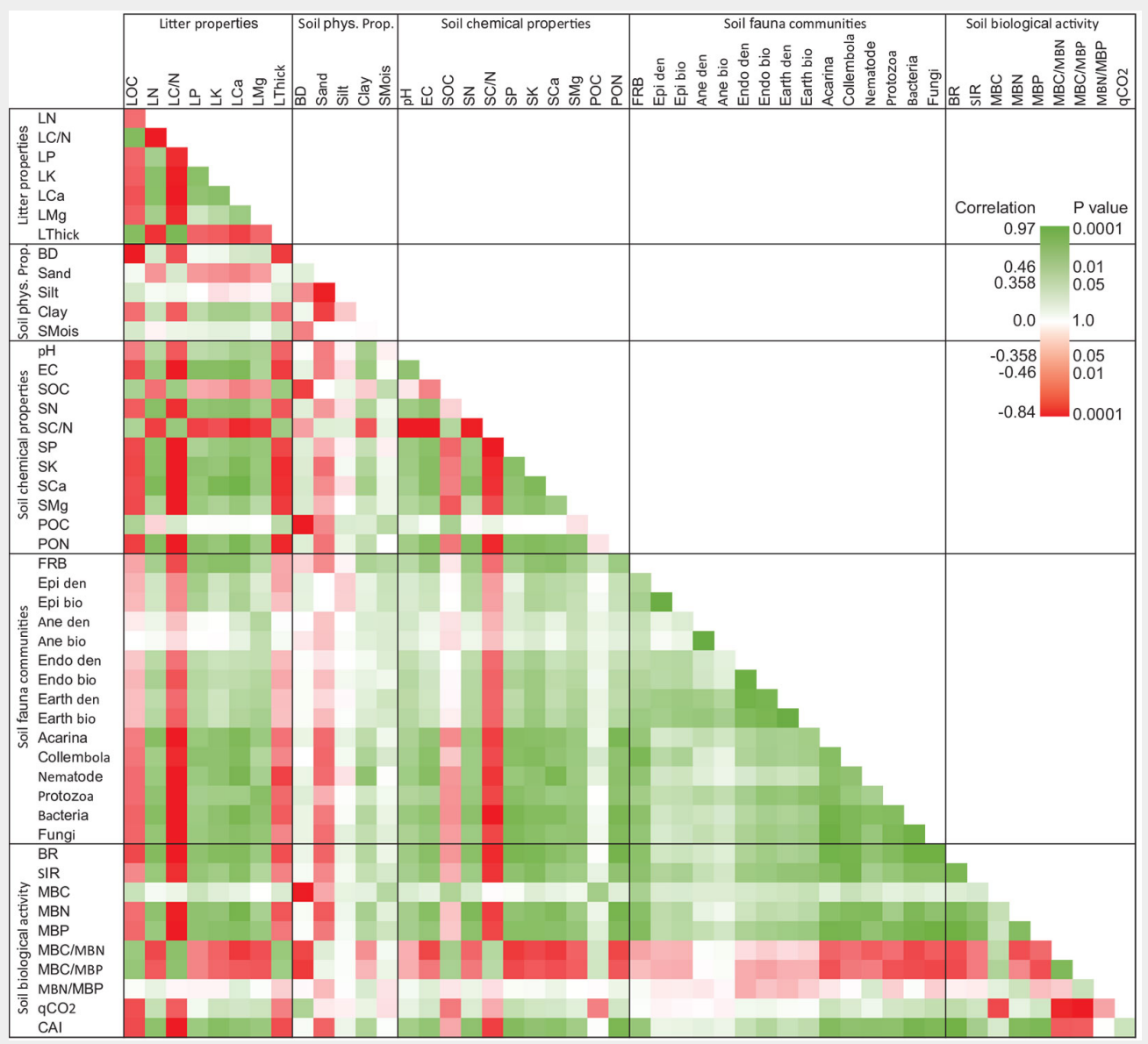

Fig. 4 - Correlation heatmap of studied soil properties $(\mathrm{N}=30)$. Variable labels are reported in the List of Abbreviations (see below). 


\section{Discussion}

\section{Litter and soil physicochemical}

\section{properties}

Litter production and decomposition are two important processes for the formation of soil organic matter and the nutrient cycle. Also, the ratio between production and decomposition determines the thickness of the substrate layer on the forest floor (León \& Osorio 2014). In this study, litter in CSP and QCP stands have a low degradation rate, which was the result of poor litter quality of CSP and high lignin content of QCP litter (Zarafshar et al. 2020), which creates a thicker organic layer under these species compared to ASP, a nitrogen-fixing species with high litter nitrogen content (Llorente et al. 2010). However, a faster decomposition under VNF and AVP forests in this study is probably due to a more attractive source for decomposers due to the lower $\mathrm{C} / \mathrm{N}$ ratio, which resulted in thinner litter layer (Walmsley et al. 2019).

Vivanco \& Austin (2019) found that litter decomposition rate is significantly controlled by tree species rather than climate, and not only macronutrients can contribute to litter decomposition, but also micronutrients have significant effect on the process. Along with litter quality, soil texture has also a significant role in litter decomposition process by providing moisture and greater microbial biomass due to higher clay content (Montes-Pulido et al. 2017). This effect can be seen in VNF, AVP, and ASP as the clay content of the forest floor was higher than other stands. Soil $\mathrm{pH}$ and EC are also dependent on the vegetation cover of the soil and the decomposition process. Haghdoost et al. (2011) found that soil EC depends on the type and properties of vegetation litter. On the other hand, lower $\mathrm{pH}$ of CSP can be due to the acidification of coniferous litter (Vázquez et al. 2020). Our study showed similar results to those of abovementioned studies in case of $\mathrm{pH}$, however, low $\mathrm{pH}$ in QCP and DNF can be due to $\mathrm{C} / \mathrm{N}$ ratio of the leaf litters. Kooijman et al. (2019) found that low litter quality in beech stands resulted in high $\mathrm{pH}$ compared to hornbeam stands. Therefore, we can assume that under highly decomposable leaf litter the $\mathrm{pH}$ value is significantly higher, which explains why the litter thickness is higher in the stands with low soil $\mathrm{pH}$ in our study (Mohr \& Topp 2005).

We observed that VNF and ASP have significantly higher amounts of available $\mathrm{P}, \mathrm{K}$, $\mathrm{Ca}$, and $\mathrm{Mg}$. This might be the result of higher soil moisture and higher biological activity of the soil microorganisms derived by higher $\mathrm{pH}$, which have led to faster decomposition of the litter layer, while keeping $\mathrm{pH}$ at suitable levels (Margenot et al. 2018).

Nitrogen availability in VNF and ASP can be attributed to the presence of nitrogen fixing tree species like $A$. subcordata. This species can increase soil nitrogen availabil- ity by increasing the activity of nitrogen-fixing microorganisms (Wang et al. 2019). On the other hand, higher $\mathrm{C} / \mathrm{N}$ ratios, lower $\mathrm{pH}$, and lower nutrient content in coniferous needles compared to broadleaves have previously been reported (Vázquez et al. 2020). POM-C and POM-N provide a sensitive indicator that reflects the impact of short-term changes in soil surface on soil quality. High levels of litter under CSP and QCP lead to significantly higher amounts of POM-C which can be the result of lower decomposition rate in this stands (Vázquez et al. 2020). On the other hand, higher amounts of nitrogen in ASP and VNF litter led to higher POM-N which is one of the characteristic of these forests (Kooch et al. 2018). Studies have shown that soil properties are affected by the interaction effect of substrate and tree species. In a study on 4 different tree species in Poland, Józefowska et al. (2017) found that soil C:N ratio depends on tree species as well as soil fauna communities. However, as our stands were close to each other, we found that soil properties are affected directly by tree species.

\section{Soil fauna community}

Fine root, earthworm, and other soil organism biomass were significantly higher in VNF and ASP compared to other forest covers. Studies on soil respiration showed that basal respiration (BR) significantly depends on soil mineralization process, which mostly results from chemical and biological reactions of the soil and also root respiration of the forest covers (Wang et al. 2020). Our results showed that both BR and SIR significantly increase from $D N F<$ $C S P<Q C P<A V P<A S P<V N F$, which indicates that low forest cover and also conifer forests have lower soil respiration. These results are in line with the results of Jílková (2020) who showed that a shift in forest cover from conifers to deciduous forests can significantly increase soil respiration. On the other hand, litter properties have significant effects on soil respiration by providing essential nutrients which are needed by soil microorganisms. In other words, in forests with higher litter quality and litter nutrient content, the rate of soil BR and SIR is significantly higher, as recorded in VNF and ASP in this study (Jílková 2020). A sharp decline in soil respiration in both SCP and DNF is the result of low biological activity in both forest covers due to lower soil pH and lower water content (Vázquez et al. 2020, Wang et al. 2020).

Forest degradation and the following decline of the canopy created an unfavorable climate for the activity of soil organisms under the degraded forest. The substrate layer is also essential for soil organisms because it acts as both habitat and food source (Tajik et al. 2020), which is scarce under DNF cover.

Increase in vegetation diversity, aboveground biomass, and forest structure tends to increase the abundance of soil organisms (Diao et al. 2020), which is the case of VNF in our study. Indeed, higher litter quality and higher soil organism biomass and activity in VNF compared to other forest cover were observed, which could explain the higher SIR. Soils under natural forest with lower bulk density, higher water and nutrients content, and low C:N ratio, with appropriate soil $\mathrm{pH}$ has significantly increased the community of soil organisms (Kooch et al. 2020). On the other hand, reforestation of the degraded forests with broadleaved species also increased soil organisms (De Oliveira Paulucio et al. 2017), which is the case of ASP and AVP in this study.

Earthworms, small arthropods (Acarina and Collembola), nematodes, protozoa, bacteria, and fungi in ASP and AVP were all significantly higher than in DNF and CSP. This can imply a higher nutrient availability of the forest soil and thinner litter layer due to higher biological activity in the soil (Elie et al. 2018). Li et al. (2019) found that a larger community of soil microorganisms can lead to faster decomposition of litter and thus higher availability of nutrients to vegetation roots. Additionally, in forests with high litter quality and low litter $\mathrm{C}: \mathrm{N}$ ratio, faunal activity (especially earthworms) is higher, which leads to higher bioturbation and thinner litter layer (Frouz et al. 2013).

Among the earthworm groups, only endogeic showed a significant increase in the following order: $D N F<C S P<Q C P<A V P$ $\angle A S P<V N F$, which may be due to the movement of endogeic groups to different layers of soil where they are able to establish in better conditions (Kooch et al. 2020). Acarina and Collembola are microphages and indirectly affect the process of the nutrient cycle by controlling the bacteria and fungi in the soil community (Wardle \& Lavelle 1997). Small arthropods and protozoa are associated with soil $\mathrm{pH}$ in forest ecosystems. The amount of nematodes often shows the highest value in soils with $\mathrm{pH} 7.0$ or higher compared to soils with $\mathrm{pH}$ between 5.9 and 6.5 (Matute 2013), which can explain our results.

\section{Soil biological activity}

MBN is an indicator of soil quality and is used as a criterion for the availability of nitrogen for plants. MBN in VNF and ASP were significantly higher than that of other forest covers, which also confirms that the tree species has affected the soil microbial community (Diao et al. 2020). Although non-significant, MBN was roughly $20 \%$ higher in ASP compared to VNF, which indicates the key role of nitrogen-fixing tree species in providing nitrogen for microbial communities, while VNF could provide significantly more $P$ to microbial communities (and higher MBP). This is contrary to what observed for MBC. CSP showed significantly higher MBC compared to ASP, which is an indicator of difference in litter input 
quality between the two reforested stands (Kooch et al. 2018). The microbial indicators $M B C / M B N$ and $M B C / M B P$ differed significantly across different forest covers. AC cording to Kooch et al. (2018), such significant changes can be attributed to the input of plant residues with different characteris tics of understory (i.e., to the annual vegetation cover on the ground) in the studied sites.

Changes in metabolic rate can indicate that different microbial community struc tures have occurred according to different tree species (Wang et al. 2019). It also can be affected by soil moisture, soil $\mathrm{pH}$, organic carbon, total nitrogen content and nutritional status of the different forest covers in the study area (Cui et al. 2020). In this regard, ASP with higher soil $\mathrm{pH}$ and $\mathrm{ni}-$ trogen content has the highest metabolic rate. To better understand the state of microbial communities, we also calculated CAI (Gorobtsova et al. 2016). This parameter which is the ratio between BR and SIR is significantly lower in DNF compared to other forest stands. Such low value for CA is the result of significantly lower $B R$ in DNF stand. While SIR in VNF is almost two times higher than that of DNF, BR is 6 times higher. This means that significantly low biological activity in DNF soils resulted in low BR (Vázquez et al. 2020).

Our results showed significant positive correlation between fauna populations, and soil CAI and respiration. This correlation can demonstrate the key role of soil fauna in litter decomposition, as they help distribute organic materials through different soil layers and help leaching and re lease of POM (Frouz 2018).

\section{Conclusion}

Our study showed that old grown natural forests are key forests to maintain maximum soil quality and microbial activity, however, some key species like $A$. subcordata can significantly revive soil quality after a short period of time. In our study, most biological and microbial indicators were significantly higher in close-to-virgin natural forest and $A$. subcordata plantations (e.g., ecological groups of earth worms, Acarina, Collembola, nematodes, protozoa, bacteria and fungi), along with soil nutrients ( $\mathrm{N}, \mathrm{P}, \mathrm{K}, \mathrm{Ca}$, and $\mathrm{Mg}$ ), which means not only vegetative intactness can contribute to higher soil quality, but also litterfall have a key role in soil activity and fertility. While the activities of soil organ isms and soil fertility were at the lowest levels in degraded forests, $C$. sempervirens plantations, and Q. castaneifolia plantations, they have been significantly in creased in $A$. subcordata plantations only after 3 decades. In addition, soil microbial activities significantly depend on litter $\mathrm{N}$ content and $\mathrm{C}: \mathrm{N}$ ratio along with soil $\mathrm{pH}$, which itself is also a dependent variable to litter quality. A. subcordata, an endemic nitrogen-fixing tree species to these forests, has a good growth rate, and plays an im- portant role in the nutrient cycle and soilrelated processes in temperate ecosystems. Therefore, it can be recommended for the restoration of degraded natural forests. Our study clearly shows that the main effect of forest degradation can be directly reflected to soil quality, soil fauna community, and biological activity. On the other hand, species selection for reforestation can significantly contribute to soil restoration, to an extent that planting $C$. sempervirens, and Q. castaneifolia does not make any difference from a degraded forest.

\section{List of abbreviations}

Acarina: Acarina density; Ane bio: Anecic biomass; Ane den: Anecic density; ASP: Alnus subcordata; AVP: Acer velutinum; Bacteria: Total bacteria; BD: Bulk density; BR: Basal respiration; CAl: Carbon availability index; Collembola: Collembola density; CSP: Cupressus sempervirens; DNF: Degraded natural forest; Earth bio: Earthworm biomass; Earth den: Earthworm density; Endo bio: Endogeic biomass; Endo den: Endogeic density; Epi bio: Epigeic biomass; Epi den: Epigeic density; FRB: Fine root biomass; Fungi: Total fungi; LC/N: Litter C/N ratio; LCa: Available litter calcium; LK: Available litter potassium; LMg: Available litter magnesium; LN: Litter nitrogen; LOC: Litter carbon; LP: Available litter phosphorus; LThick: Litter thickness; MBC: Microbial biomass of carbon; MBN: Microbial biomass of nitrogen; MBP: Microbial biomass of phosphorus; Nematode: Total nematode; POM-C: Particulate organic matter carbon; POM-N: Particulate organic matter nitrogen; Protozoa: Protozoa density; $\mathrm{qCO}_{2}$ : Metabolic quotient; QCP: Quercus castaneifolia; SCa: Available soil calcium; $\mathrm{SC} / \mathrm{N}$ : Soil C/N ratio; SIR: Substrate induced respiration; SK: Available soil potassium; SMg: Available soil magnesium; SMois: Soil moisture; SN: Soil total nitrogen; SOC: Soil organic carbon; SP: Available soil phosphorus; VNF: Close-to-virgin natural forest.

\section{Acknowledgments}

M.B. and A.S. carried out the field measurements; Y.K. performed the statistical analysis; V.E. and M.B. performed the laboratory analysis; M.B. conceived the study and helped to draft the manuscript. The authors wish to thank Mr. Jahansouz Foroughi for his helps on field sampling and Mr. Ehsan Khedive for his assistance on writing the manuscript. This work was supported by financial supports of the University of Tehran, Iran.

\section{References}

Alef K (1995). Estimating of soil respiration. In: "Methods in Soil Microbiology and Biochemistry" (Alef K, Nannipieri $P$ eds). Academic Press, New York, USA, pp. 464-470.

Allison LE (1965). Organic carbon. In: “Methods of Soil Analysis. Part 2. Chemical and Microbiological Properties" (Page AL, Miller RH, Keeney DR eds). American Society of Agronomy, Soil
Science Society of America, Madison, WI, USA, pp. 1367-1378. - doi: 10.2134/agronmonogr9.2. C39

Anderson T-H, Domsch KH (1990). Application of eco-physiological quotients $\left(\mathrm{qCO}_{2}\right.$ and $\left.\mathrm{qD}\right)$ on microbial biomasses from soils of different cropping histories. Soil Biology and Biochemistry 22: 251-255. - doi: 10.1016/0038-0717(90)90 094-G

Bouyoucos GJ (1962). Hydrometer method improved for making particle size analysis of soils. Journal of Agrobiology 56: 464-465. - doi: 10.2134/agronj1962.00021962005400050028x Bremner JM, Mulvaney CS (1982). Nitrogen-total. In: "Methods of Soil Analysis. Part 2. Chemical and Microbiological Properties" (Page AL, Miller RH, Keeney DR eds). American Society of Agronomy, Soil Science Society of America, Madison, WI, USA, pp. 595-624.

Brookes PC, Landman A, Pruden G, Jenkinson DS (1985). Chloroform fumigation and the release of soil nitrogen: a rapid direct extraction method to measure microbial biomass nitrogen in soil. Soil Biology and Biochemistry 17: 837-842. doi: 10.1016/0038-0717(85)90144-0

Cheng W, Zhang Q, Coleman DC, Carroll CR, Hoffman CA (1996). Is available carbon limiting microbial respiration in the rhizosphere? Soil Biology and Biochemistry 28: 1283-1288. - doi: 10.1016/S0038-0717(96)00138-1

Cui Y, Wang X, Zhang X, Ju W, Duan C, Guo X, Wang Y, Fang L (2020). Soil moisture mediates microbial carbon and phosphorus metabolism during vegetation succession in a semiarid region. Soil Biology and Biochemistry 147: 107814. - doi: 10.1016/j.soilbio.2020.107814

De Oliveira Paulucio V, Da Silva CF, Martins MA, Pereira MG, Schiavo JA, Rodrigues LA (2017). Reforestation of a degraded area with Eucalyptus and Sesbania: microbial activity and chemical soil properties. Revista Brasileira de Ciencia do Solo 41: e0160239. - doi: 10.1590/18069657rb cs20160239

Diao M, Yang K, Zhu J, Li M, Xu S (2020). Native broad-leaved tree species play key roles on maintaining soil chemical and microbial properties in a temperate secondary forest, Northeast China. Forest Ecology and Management 462: 117971. - doi: 10.1016/j.foreco.2020.117971

Edwards CA, Arancon NQ, Dumanig F (2021). Biology and ecology of earthworms. Springer Science and Business Media, USA, pp. 422. [online] URL: http://books.google.com/books?id= ad4rDwD GhsC

Elie F, Vincenot L, Berthe T, Quibel E, Zeller B, Saint-André L, Normand $M$, Chauvat $M$, Aubert $M$ (2018). Soil fauna as bioindicators of organic matter export in temperate forests. Forest Ecology and Management 429: 549-557. - doi: 10.10 16/j.foreco.2018.07.053

Frouz J, Livečková M, Albrechtová J, Chronáková A, Cajthaml T, Pizl V, Hánel L, Stary J, Baldrian P, Lhotáková Z, Simáčková H, Cepáková S (2013). Is the effect of trees on soil properties mediated by soil fauna? A case study from postmining sites. Forest Ecology and Management 309: 87-95. - doi: 10.1016/j.foreco.2013.02.013

Frouz J (2018). Effects of soil macro- and mesofauna on litter decomposition and soil organic matter stabilization. Geoderma 332: 161-172. doi: 10.1016/j.geoderma.2017.08.039 
Gorobtsova ON, Gedgafova FV, Uligova TS, Tembotov RK (2016). Ecophysiological indicators of microbial biomass status in chernozem soils of the Central Caucasus (in the territory of Kabardino-Balkaria with the Terek variant of altitudinal zonation). Russian Journal of Ecology 47: 19-25. - doi: 10.1134/S1067413616010069

Haghdoost N, Akbarinia M, Hosseini SM, Kooch $Y$ (2011). Conversion of Hyrcanian degraded forests to plantations: effects on soil $\mathrm{C}$ and $\mathrm{N}$ stocks. Annals of Biological Research 2: 385399. [online] URL: http://www.researchgate. net/publication/275333499

Handayani IP, Coyne MS, Tokosh RS (2010). Soil organic matter fractions and aggregate distribution in response to tall fescue stands. International Journal of Soil Science 5: 1-10. - doi: 10.3923/ijss.2010.1.10

Homer CD, Pratt PF (1961). Methods of analysis for soils, plants and waters. Agricultural Sciences Publications, University of California, Berkeley, CA, USA, pp. 309.

Hutson BR, Veitch LG (1987). Densities of Collembola and Acarina in the soil and litter of three indigenous South Australian forests related to layer, site and seasonal differences. Australian Journal of Ecology 12: 239-261. - doi: 10.1111/j.144 2-9993.1987.tboog46.x

Jiang J-P, Xiong Y-C, Jiang H-M, De-You YE, Ya-Jie $S$, Feng-Min LI (2009). Soil microbial activity during secondary vegetation succession in semiarid abandoned lands of Loess Plateau. Pedosphere 19: 735-747. - doi: 10.1016/S1002-0160 (09)60169-7

Jílková V (2020). Soil respiration in temperate forests is increased by a shift from coniferous to deciduous trees but not by an increase in temperature. Applied Soil Ecology 154: 103635. - doi: 10.1016/j.apsoil.2020.103635

Józefowska A, Pietrzykowski M, Cajthaml T, Frouz J (2017). The effects of tree species and substrate on carbon sequestration and chemical and biological properties in reforested postmining soils. Geoderma 292: 9-16. - doi: 10.1016/ j.geoderma.2017.01.008

Kooch Y, Tavakoli M, Akbarinia M (2018). Microbial/biochemical indicators showing perceptible deterioration in the topsoil due to deforestation. Ecological Indicators 91: 84-91. - doi: 10.101 6/j.ecolind.2018.03.084

Kooch Y, Bayranvand M (2019). Labile soil organic matter changes related to forest floor quality of tree species mixtures in Oriental beech forests. Ecological Indicators 107 (6): 105598. - doi: 10.1016/j.ecolind.2019.105598

Kooch Y, Ehsani S, Akbarinia M (2020). Stratification of soil organic matter and biota dynamics in natural and anthropogenic ecosystems. Soil and Tillage Research 200: 104621. - doi: 10.1016/ j.still.2020.104621

Kooijman AM, Weiler HA, Cusell C, Anders N, Meng $X$, Seijmonsbergen AC, Cammeraat LH (2019). Litter quality and microtopography as key drivers to topsoil properties and understorey plant diversity in ancient broadleaved forests on decalcified marl. Science of the Total Environment 684: 113-125. - doi: 10.1016/j.scitot env.2019.05.285

León JD, Osorio NW (2014). Role of litter turnover in soil quality in tropical degraded lands of Colombia. The Scientific World Journal 2014: 693981. - doi: 10.1155/2014/693981

Li X, Dong W, Song Y, Wang W, Zhan W (2019). Effect of soil fauna on home-field advantages of litter mass loss and nutrient release in different temperate broad-leaved forests. Forests 10 (11): 1033. - doi: 10.3390/f10111033

Liang W, Lou Y, Li Q, Zhong S, Zhang X, Wang J (2009). Nematode faunal response to longterm application of nitrogen fertilizer and organic manure in Northeast China. Soil Biology and Biochemistry 41: 883-890. - doi: 10.1016/j. soilbio.2008.06.018

Llorente M, Glaser B, Turrión MB (2010). Storage of organic carbon and black carbon in density fractions of calcareous soils under different land uses. Geoderma 159: 31-38. - doi: 10.1016/j. geoderma.2010.06.011

Margenot AJ, Sommer R, Parikh SJ (2018). Soil phosphatase activities across a liming gradient under long-term managements in Kenya. Soil Biology and Biochemistry 82: 850-861. - doi: 10.2136/sssaj2017.12.0420

Matute MM (2013). Soil nematodes of Brassica rapa: influence of temperature and $\mathrm{pH}$. Advances in Natural Science 6: 20-26. [online] URL: http://52.196.142.242/index.php/ans/article /view/j.ans.1715787020130604.2858

Mayzlish E, Steinberger Y (2004). Effects of chemical inhibitors on soil protozoan dynamics in a desert ecosystem. Biology and Fertility of Soils 39: 415-421. - doi: 10.1007/s00374-004-072 3-9

McCune B, Mefford MJ (1999). PC-ORD: multivariate analysis of ecological data, version 4 . MjM software design, Gleneden Beach, OR, USA.

Mohr D, Topp W (2005). Hazel improves soil quality of sloping oak stands in a German low mountain range. Annals of Forest Science 62: 23-29. - doi: 10.1051/forest:2004090

Montes-Pulido CR, Ramos-Miras JJ, José-Wery S, María A (2017). Estimation of soil organic carbon (SOC) at different soil depths and soil use in the Sumapaz paramo, Cundinamarca-Colombia. Acta Agronómica 66: 95-101. - doi: 10.15446/ acag.v66n1.53171

Nave LE, Walters BF, Hofmeister KL, Perry $\mathrm{CH}$, Mishra U, Domke GM, Swanston CW (2019). The role of reforestation in carbon sequestration. New Forests 50: 115-137. - doi: 10.1007/s11 056-018-9655-3

Plaster EJ (2013). Soil science and management. Cengage Learning, Albany, NY, USA, pp. 544.

Sanji R, Kooch Y, Rey A (2020). Impact of forest degradation and reforestation with Alnus and Quercus species on soil quality and function in northern Iran. Ecological Indicators 112 (7): 106 132. - doi: 10.1016/j.ecolind.2020.106132

Shao P, Liang C, Lynch L, Xie H, Bao X (2019). Reforestation accelerates soil organic carbon accumulation: evidence from microbial biomarkers. Soil Biology and Biochemistry 131: 182-190. doi: 10.1016/j.soilbio.2019.01.012
Soil Survey Staff (2002). Soil taxonomic maps of northern Iran. Soil and Water Research Institute, Alborz, Iran, pp. 234.

Tajik S, Ayoubi S, Lorenz N (2020). Soil microbial communities affected by vegetation, topography and soil properties in a forest ecosystem. Applied Soil Ecology 149 (306): 103514. - doi: 10.1016/j.apsoil.2020.103514

Tavakoli M, Kooch Y, Akbarinia M (2018). The effect of degraded and reclaimed forest areas on carbon dioxide gas emissions and soil carbon mineralization in West of Mazandaran. In: Proceedings of the "International Symposium of Climate Change and Dendrochronology in Caspian Ecosystems". Sari (Iran), 16-17 May 2008, pp. 12.

Vázquez $\mathrm{E}$, Benito $\mathrm{M}$, Espejo R, Teutscherova $\mathrm{N}$ (2020). Response of soil properties and microbial indicators to land use change in an acid soil under Mediterranean conditions. Catena 189: 104486. - doi: 10.1016/j.catena.2020.104486

Vivanco L, Austin AT (2019). The importance of macro- and micro-nutrients over climate for leaf litter decomposition and nutrient release in Patagonian temperate forests. Forest Ecology and Management 441: 144-154. - doi: 10.1016/j. foreco.2019.03.019

Walmsley A, Vachová P, Hlava J (2019). Tree species identity governs the soil macrofauna community composition and soil development at reclaimed post-mining sites on calcium-rich clays. European Journal of Forest Research 138: 753-761. - doi: 10.1007/s10342-019-01202-5

Wang $\mathrm{H}$, Liu S, Song $Z$, Yang $Y$, Wang J, You $Y$, Zhang $X$, Shi Z, Nong $Y$, Ming A, Lu L, Cai D (2019). Introducing nitrogen-fixing tree species and mixing with Pinus massoniana alters and evenly distributes various chemical compositions of soil organic carbon in a planted forest in southern China. Forest Ecology and Management 449: 117477. - doi: 10.1016/j.foreco.2019.117 477

Wang Y, Liu S, Wang J, Chang SX, Luan J, Liu Y, Lu H, Liu X (2020). Microbe-mediated attenuation of soil respiration in response to soil warming in a temperate oak forest. Science of The Total Environment 711 (7): 134563. - doi: 10.1016/ j.scitotenv.2019.134563

Wardle DA, Lavelle P (1997). Linkages between soil biota, plant litter quality and decomposition. In: "Driven by Nature: Plant Litter Quality and Decomposition" (Gadish G, Giller KE eds). CAB International, Wallingford, UK, pp. 107-124. Wollum AG (1983). Cultural methods for soil microorganisms. In: "Methods of Soil Analysis. Part 2. Chemical and Microbiological Properties" (Page AL, Miller RH, Keeney DR eds). American Society of Agronomy, Soil Science Society of America, Madison, WI, USA, pp. 781802. - doi: 10.2134/agronmonogr9.2.2ed.c37

Zarafshar M, Bazot S, Matinizadeh M, Bordbar SK, Rousta MJ, Kooch Y, Enayati K, Abbasi A, Negahdarsaber M (2020). Do tree plantations or cultivated fields have the same ability to maintain soil quality as natural forests? Applied Soil Ecology 151 (12): 103536. - doi: 10.1016/j.ap soil.2020.103536 